\title{
Vogelpohl, Anne; Michel, Boris; Lebuhn, Henrik; Hoerning, Johanna; Belina, Bernd (Hrsg.) (2018): Raumproduktionen II. Theoretische Kontroversen und politische Auseinandersetzungen.
}

\author{
Münster: Westfälisches Dampfboot. = Raumproduktionen, Band 26. 191 Seiten
}

\section{Sebastian Schipper ${ }^{1}$ iD}

Eingegangen: 6. Juli 2018 / Angenommen: 19. Juli 2018 / Online publiziert: 7. August 2018

(c) Springer-Verlag GmbH Deutschland, ein Teil von Springer Nature 2018

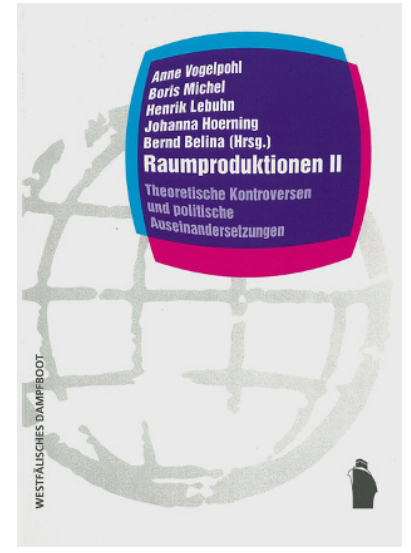

Im Jahr 2007 haben Bernd Belina, Boris Michel und Markus Wissen die Reihe „Raumproduktionen“ beim Verlag Westfälisches Dampfboot mit dem Ziel eingeführt, Positionen und Argumente der angelsächsischen Radical Geography und kritischer raumbezogener Forschung innerhalb der deutschsprachigen Geographie sowie in benachbarten Sozialwissenschaften bekannter zu machen. Ausgehend von dem gleichnamigen Pionierband, der zentrale Texte aus der englischsprachigen Debatte erstmalig in deutscher Übersetzung enthält, sind in der Reihe mittlerweile 31 weitere Bände erschienen. Basierend auf der Grundannahme, dass Gesellschaft durch und durch räumlich konstituiert und Raum zugleich ein Produkt gesellschaftlicher Praxis ist, leisten die in der Buchreihe veröffentlichten Dissertationen und

Dr. habil. Sebastian Schipper

S.Schipper@geo.uni-frankfurt.de

1 Institut für Humangeographie, Goethe-Universität Frankfurt a.M., Theodor-W.-Adorno-Platz 6, 60629 Frankfurt am Main, Deutschland
Sammelbände allesamt einen spezifischen Beitrag, um die räumliche Dimension von Gesellschaft als „Komplex aus Gebautem, Natur, politischen Verhältnissen, wirtschaftlichen Beziehungen, Diskursen, Wünschen und alltäglichem Leben“ (S. 7) besser verstehen zu können.

Als übergreifende Spezifika zeichnet die Reihe aus, dass sie erstens auf theoretischer Ebene strukturelle Rahmenbedingungen in den Blick nimmt, zweitens praxisorientiert auf konkrete Lebensverhältnisse fokussiert und drittens den emanzipatorischen Anspruch verfolgt, einen Beitrag zur Überwindung gesellschaftlich eingerichteter Machtverhältnisse leisten zu wollen. Erfolgreich ist die Buchreihe insofern, da sie entscheidend daran mitgewirkt hat, dass es mittlerweile einen ,,eigenständige[n] deutschsprachige[n] Diskussionszusammenhang zu Fragen, Positionen und Themen der Radical Geography“ (S. 9) gibt. Konsequenterweise will der Jubiläumsband „Raumproduktionen II“, der nun zum zehnjährigen Bestehen der Reihe erschienen ist, daher auch keine Übersetzungsarbeit mehr leisten, sondern ,zur Schärfung eines explizit kritischen und materialistisch konturierten Zugangs zu Raumproduktionen beitragen“ (S. 9).

Gerahmt von einer Einleitung und einem Resümee der Herausgeberinnen und Herausgeber ist der Sammelband entlang von acht zentralen Gegenständen organisiert: Infrastrukturen als soziotechnische Netzwerke (Stefan Höhne/ Matthias Naumann), Körper als machtgeladene Intra-aktionen zwischen Biologischem und Sozialem (Nadine Marquardt/Anke Strüver), Umwelt und die neue Einhegung der Natur (Maria Backhouse), Planung und Partizipation (Nina Gribat/Manuel Lutz), Protest aus der Perspektive kritischer Gesellschaftsforschung (Catarina Gomes de Matos/Daniel Mullis), Urban Citizenship und Recht auf Stadt (Henrik Lebuhn), Algorithmen und Big Data (Jan Kemper/Till Straube) sowie Städte aus Sicht eines postkolonial-materialistischen Ansatzes (Johanna Hoerning). Die Auswahl der 
Themen zeichnet sich dadurch aus, dass sie zum einen aktuelle gesellschaftliche Konfliktfelder in sich bündeln und zum anderen zentrale Begrifflichkeiten neuerer Theorieangebote darstellen. Letzteres ist wesentlich, da der Band das übergreifende Ziel verfolgt, in jedem Kapitel bereits etablierte kritisch-materialistische Theorietraditionen mit einem aktuellen Theorieangebot, wie etwa der Actor-Network Theory (ANT) oder dem „Neuen Materialismus“, in Dia$\log$ zu bringen. Dadurch sollen die jeweiligen Stärken der Theorien für die Analyse ungleicher räumlicher Verhältnisse benannt, aber auch auf beiden Seiten Leerstellen und fruchtbare Ergänzungen herausgearbeitet werden. Bemerkenswert ist, dass es den Herausgeberinnen/Herausgebern und Autorinnen/Autoren gelungen ist, ansonsten häufig zu beobachtende ,scholastische Streitigkeiten“ über die ,,,besseren " und ,schlechteren" Theorien“ (S. 11) zu vermeiden, indem bewusst politisch brisante Gegenstände ausgewählt und konsequent gesellschaftliche Praktiken in den Mittelpunkt der Auseinandersetzungen gestellt worden sind.

In ihrem Resümee des Sammelbandes halten Johanna Hoerning und Henrik Lebuhn fest, dass die Spannungen zwischen kritisch-materialistischen Gesellschaftstheorien und neueren Theorieangeboten, die häufig in expliziter Abgrenzung zum kritischen Materialismus begründet worden sind, notwendigerweise bestehen bleiben mussten. Dass sich die unterschiedlichen Ansätze nicht umstandslos zusammenführen und die divergenten Grundannahmen nicht widerspruchslos aufheben lassen, verdeutlicht sehr anschaulich der Beitrag von Jan Kemper und Till Straube zu Algorithmen und Big Data. Ihren Gegenstand betrachten die beiden Autoren einerseits aus politökonomischer Sicht als Ausdruck einer Produktion von relativem Mehrwert und als Strategie zur Abschöpfung einer Informationsrente im Kapitalverwertungsprozess, andererseits aus der Mikroperspektive der Actor-Network Theory und der Science and Technology Studies (STS) als kleinteilige soziotechnische Konfiguration, die einer detaillierten Beschreibung und empirischen Durchdringung bedarf. Angesichts der theoretisch-konzeptionellen Unvereinbarkeit beider Ansätze, die entweder in Marx'scher Tradition auf strukturalistische Erklärungen abzielen oder im Sinne von ANT und STS auf dichte Deskriptionen fokussieren, sei eine Synthese ,weder machbar noch nötig“ (S. 154). Möglich wäre aber zumindest eine polysemische Lesart der Gegenstände, welche deren systematische Mehrdeutigkeit aufzeigt, die jeweiligen Stärken der beiden Theorieansätze betont und den Dialog mit der jeweils anderen Perspektive immerhin offenhält.

Dank dieser Offenheit, die auch sämtliche andere Artikel durchzieht, gelingt es in dem Sammelband trotz mancher offenkundiger theoretisch-konzeptioneller Unvereinbarkeiten, produktive Überschneidungen und Synergieeffekte zu identifizieren, die der kritisch-materialistischen Raumfor- schung im Ergebnis neue Impulse verleihen. Wie Hoerning und Lebuhn in ihrem Fazit überzeugend herausarbeiten, erweitern und schärfen die versammelten Beiträge insgesamt die kritisch-materialistische Theoriebildung, indem sie gegenständliche Materialitäten, subjektive Sinnkonstruktionen, diskursive Rahmungen sowie situative und verkörperte Praktiken auf theoriebildender Ebene miteinbeziehen.

Allerdings entstünden dadurch zugleich auch kontraproduktive ,theoretische Fliehkräfte“ (S. 183), die insbesondere, aber nicht nur beim Assemblage-Ansatz sichtbar würden. Derartige Arbeiten gingen häufig mit der Tendenz einher, ,sich immer weiter von den Eckpfeilern kritisch-materialistischer Gesellschaftsanalyse“ zu entfernen und ,sich Fragen von Herrschafts- und Machtstrukturen oder auch sozialer Ungleichheit zu entziehen“ (S. 183), da strukturelle Erklärungsmöglichkeiten vernachlässigt und politökonomische Fragen von kapitalistischen Eigentums- und Arbeitsverhältnissen ausgeblendet würden. Um dem entgegenzuwirken, plädieren die Herausgeberinnen und Herausgeber für eine vorsichtige Perspektivverschiebung hin zu Dingen, Körpern, Subjektivierungen und Diskursen, welche jedoch weiterhin explizit daran festhält, grundlegende Konzepte und Begriffe gesellschaftstheoretisch im Hinblick auf Macht, Herrschaft und Ungleichheit zu denken.

Ganz im Sinne des normativen Anspruchs kritisch-materialistischer Raumforschung betonen auch die Herausgeberinnen und Herausgeber, dass sie mit dem Sammelband ,nicht nur zu einem besseren Verständnis sozialräumlicher Dimensionen von Macht und Herrschaft" einen Beitrag leisten wollen, ,sondern auch zur Entwicklung politischer Ansätze, die eben diese vermachteten Verhältnisse überwinden“ (S. 187). Während sich viele Texte dementsprechend positiv auf emanzipatorisch-politische Auseinandersetzungen beziehen und eine ,,aktiv solidarische Praxis“ (S. 113) einfordern, merken Nina Gribat und Manuel Lutz in ihrem Artikel „Planung und Partizipation“ zu Recht an, dass kritische Wissenschaft ,,ihre Praxisrelevanz zumeist auf einen gut fundierten empörten Aufschrei oder einen mehr oder weniger radikal-vagen Appell an Verwaltung, Politik und Wirtschaft“" (S. 87) beschränkt. Den beiden Autoren ist daher zuzustimmen, dass sich eine kritisch-materialistische Raumforschung auch stärker ,,an die Schnittstellen der Praxis“ (S. 92) begeben muss, will sie dem vielfach formulierten eigenen emanzipatorischen Anspruch tatsächlich gerecht werden. Notwendig scheint dazu eine Reflexion der Forschungspraktiken kritischer Wissensproduktion sowie eine Überwindung der häufig zu beobachtenden Distanz zwischen akademischem Betrieb auf der einen Seite und sozialen Bewegungen, Gewerkschaften und zivilgesellschaftlichen Initiativen auf der anderen. Eine stärker anwendungsorientierte Praxis kritischer Raumforschung könnte etwa ausgehend von der Lebenswirklichkeit subalterner Gruppen Probleme aus der Praxis zivilgesell- 
schaftlicher Akteure aufgreifen und in einer dialogisch ausgerichteten Form der Wissensproduktion bearbeiten, sodass sich in der Konsequenz womöglich politische Handlungsoptionen ergeben. Während es den Herausgeberinnen und Herausgebern also eindeutig gelungen ist, notwendige und überfällige Theoriedebatten auf der Höhe der Zeit anzustoßen und die kritisch-materialistische Raumproduktion da- durch wesentlich zu schärfen, kommt der selbst formulierte Anspruch, politische Handlungsspielräume in emanzipatorischer Absicht zu öffnen, in dem ansonsten lesenswerten und erkenntnisreichen Sammelband etwas zu kurz. Hier hätte sich der Rezensent mehr Selbstkritik, Tiefenschärfe und konkretere Anregungen gewünscht. 